\title{
Nonlinear Optical Spatial Filtering for Medical Image Processing
}

\author{
Deyari Henari ${ }^{1}$, Fryad Z. Henari ${ }^{2}$ \\ ${ }^{1}$ Bahrain Defense Force Hospital, Riffa, Kingdom of Bahrain \\ ${ }^{2}$ Department of Medical Sciences, Royal College of Surgeons in Ireland, Medical University of Bahrain, \\ Busaiteen, Kingdom of Bahrain \\ Email: fzhenari@rcsi-mub.com
}

Received 7 June 2016; accepted 9 July 2016; published 12 July 2016

Copyright (C) 2016 by authors and Scientific Research Publishing Inc.

This work is licensed under the Creative Commons Attribution International License (CC BY).

http://creativecommons.org/licenses/by/4.0/

(c) (i) Open Access

\section{Abstract}

The nonlinear properties of Tris(acetylacetonato) Manganese(III) are used to manipulate the spatial frequencies at the Fourier plane using $4 \mathrm{f}-\mathrm{z}$ scan. The technique is a simple self-adaptive alloptical system, which performs image processing and nonlinear optical measurements at the same time. Preferred spatial frequencies can be selected by shifting the nonlinear sample through the focus. Edge enhancement was demonstrated by filtering of low frequency with the nonlinear material at the Fourier plane.

\section{Keywords}

Optical Nonlinearities, 4f-Z Scan, Fourier Optics, Optical Imaging

\section{Introduction}

The Z scan technique [1] has been widely used for characterization of materials exhibiting nonlinear optical effects. These effects are of great technological importance for use in future applications within electronic and photonic devices phase contrast, imaging and spatial filtering [2]-[6]. An example of interest that has attracted a lot of attention, is the light-induced optical property changes which are separated into light-induced absorption changes and light-induced refractive index changes: The light-induced absorption changes are commonly described by $\alpha=\alpha_{o}+\beta I$, where $\alpha_{o}$ is linear absorption coefficient, $I$ is the intensity of the light and $\beta$ is a nonlinear absorption coefficient. This coefficient contains interesting nonlinear optical effects such as: reverse saturation absorption (RSA), two photon absorption (TPA), and saturation absorption (SA). Both RSA and TPA have been well studied for optoelectronic and photonic devices [2] [3], optical power limiting [4], phase contrast imaging, and phase filtering [5]-[7]. The light-induced refractive index changes are described by the relationship $n=n_{o}+$ 
$n_{2} I$, where $n_{o}$ is the linear refractive index, $I$ is the intensity of the light and $n_{2}$ is a nonlinear refractive index coefficient. This coefficient is an effective parameter that contains many interesting nonlinear optical effects, such as laser-induced grating, soliton pulse propagation in waveguides [8] [9], optical switching [10]-[13], self-focusing, self-defocusing, self-phase modulation, self diffraction, optical bistability and optical limiting [14].

Nonlinear optical properties of the materials can be used for spatial filtering and medical imaging [6] [7]. Nonlinear processes such as two photon absorption, reverse saturation absorption (optical limiting), and self focusing are examples of intensity dependent transmission, where at low input intensities the transmission is linear and at high intensities the transmission is reduced. Therefore the nonlinear transmission of materials can be used for spatial filtering the undesired band in the Fourier spectrum of the image.

Optical spatial filtering using nonlinear optical materials has become very popular for implantation in optical information processing [15] such as edge enhancement and medical image processing [5] [6] [16]. A Fourier plan of the lens contains terms including: spatial frequency, the magnitude (positive and negative) and the phase. These values capture all information regarding two dimensional images at the Fourier plane. The spatial frequency is the frequency across the space that can be mapped out to the different spatial frequencies to different points in the focal plane in a 4f-image system with the nonlinear material at the Fourier plane. Therefore the Fourier spectrum contains low spatial frequencies at the center and high spatial frequency at the edge. Therefore intensity dependent nonlinear absorption can be used to filter out undesired spatial frequency bands in the Fourier spectrum of the image (low spatial frequencies at the center with high intensities and low spatial frequencies at the edges with low intensities). Spatial filtering with nonlinear optical materials has been demonstrated by many authors, Xuan et al. used two photon absorption and Raman scattering in nonlinear material such as acetone and $\mathrm{CS}_{2}$ for contrast improvement [17]. C. S. Yelleswarapu et al. [6] demonstrated the use of power limiting mechanism for self-adaptive, all optical Fourier imaging processes. Kothapalli et al. used nonlinear filtering technique that exploited photo control light modulation characteristic of bacteriorhodospin (bR) films for early detection of microcalcifications [18].

This work demonstrates the use of nonlinear absorption properties of Tris(acetylacetonato) Manganese (III) solution for all-optical Fourier image processing and filtering spatial frequencies exploiting using 4-f filtering z scan technique. The theoretical back ground for this technique is given in references [6] [19]. The Z scan technique relies on the fact that the intensity varies along the axis of the convex lens and is maximum at the focus. Hence by incorporating a nonlinear material at the Fourier plane, the desired spatial filtering component in the Fourier transformation of the object can be selected by shifting the sample through focus. The technique is used for image edge enhancement and to identify red blood cells for normal individuals and for sickle cell patients, which opens the possibility of using the technique for sickle cell diagnosis.

\section{Experimental Setup}

Figure 1 shows the experimental set up (4-f $\mathrm{z}$ scan setup) for nonlinear spatial filtering image processing. An air-cooled $\mathrm{Ar}$ ion laser beam operating at $514 \mathrm{~nm}$ with a power range of $20 \mathrm{~mW}$ is used for this study. The beam is collimated by a lens to illuminate the object and then transformed to a Fourier plane by a lens of $10 \mathrm{~cm}$ focal length $\left(\mathrm{L}_{1}\right)$. Tris(acetylacetonato) manganese(III) [Mn(acac)3] solution of $1 \mathrm{~g} / \mathrm{l}$ was prepared in acetylacetone as a sample and placed in $1 \mathrm{~mm}$ glass cuvette. The cuvette is placed at the Fourier plane for real-time processing of spatial frequency information contained in the object. The inverse Fourier transformation of the filtered spectrum is obtained using another $10 \mathrm{~cm}$ focal length lens $\left(\mathrm{L}_{2}\right)$. The transmitted beam from the sample divided into

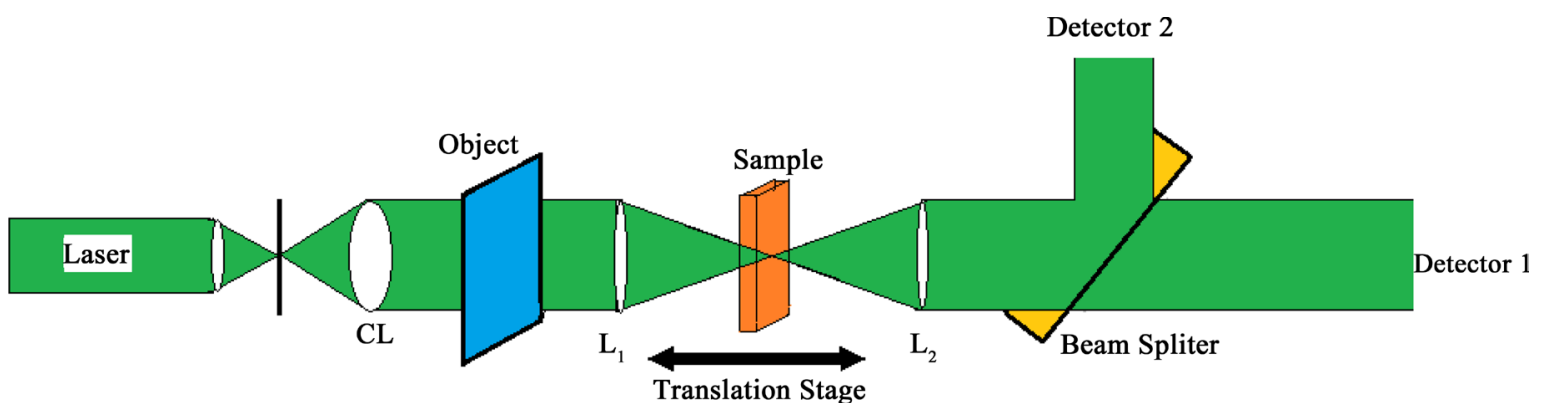

Figure 1. Experimental set up used to observe the edge enhancement using $\mathrm{Cw}$ laser at $514 \mathrm{~nm}$. 
two parts, one part of the transmitted beam is imaged on a screen at the back of the lens $L_{2}$ and the other part is detected by the detector to obtain nonlinear properties of the sample. By shifting the sample through the focus, the beam intensity and/or spatial profile is recorded. These variations of the intensity during z scan can be used to block different spatial frequency bands of the Fourier spectrum of the image. The continuous band blocking filtering can be achieved by controlling the movement of the sample through the focus of the laser beam. Therefore no neutral density filters are needed.

\section{Results and Discussion}

Firstly the object was removed (see Figure 1) and a normal Z-scan was performed to measure the nonlinear absorption and nonlinear refractive index of the sample. The transmission from the sample was measured with and without an aperture in the far-field of the lens as the sample moved through the focal point. This enables the nonlinear refractive index (closed aperture) to be separated from that of the nonlinear absorption (open aperture).

Figure 2 shows the normalizing transmission for closed aperture case. The peak valley configuration (peak then valley) of the Z-scan data indicates that the sign of the nonlinear refractive index is negative (self-defocusing). The values for nonlinear refractive index for Tris(acetylacetonato) Manganese(III) solution are reported in [20].

The nonlinear refractive index arises from local variation of the refractive index with temperature. The absorption of the focused beam propagating through the sample leads to a spatial variation of temperature in the sample and, consequently spatial variation of refractive index that acts as thermal lens resulting in the phase distortion of the beam. Figure 2 shows photographs of the variation of the spot size as a function of the sample position relative to the lens focal point: prefocus transmittance maximum (Figure 2(a)), postfocus transmittance minimum (Figure 2(c)).

At the focus where the intensity is high, diffraction rings are formed due to the self-phase modulation of a continuous wave laser beam propagating through Tris(acetylacetonato) Manganese(III) in solution (Figure 2(b)). The self-phase modulation of the laser beam is the result of the interaction between the nonlinear response of the sample with intensity dependence on refractive index and the divergence of the beam (self defocusing). This has been observed in several materials, such as atomic vapours, liquid crystals, polymers, and nanostructured materials. It was found that the number of the rings increases with increasing intensity, the higher number of observed rings

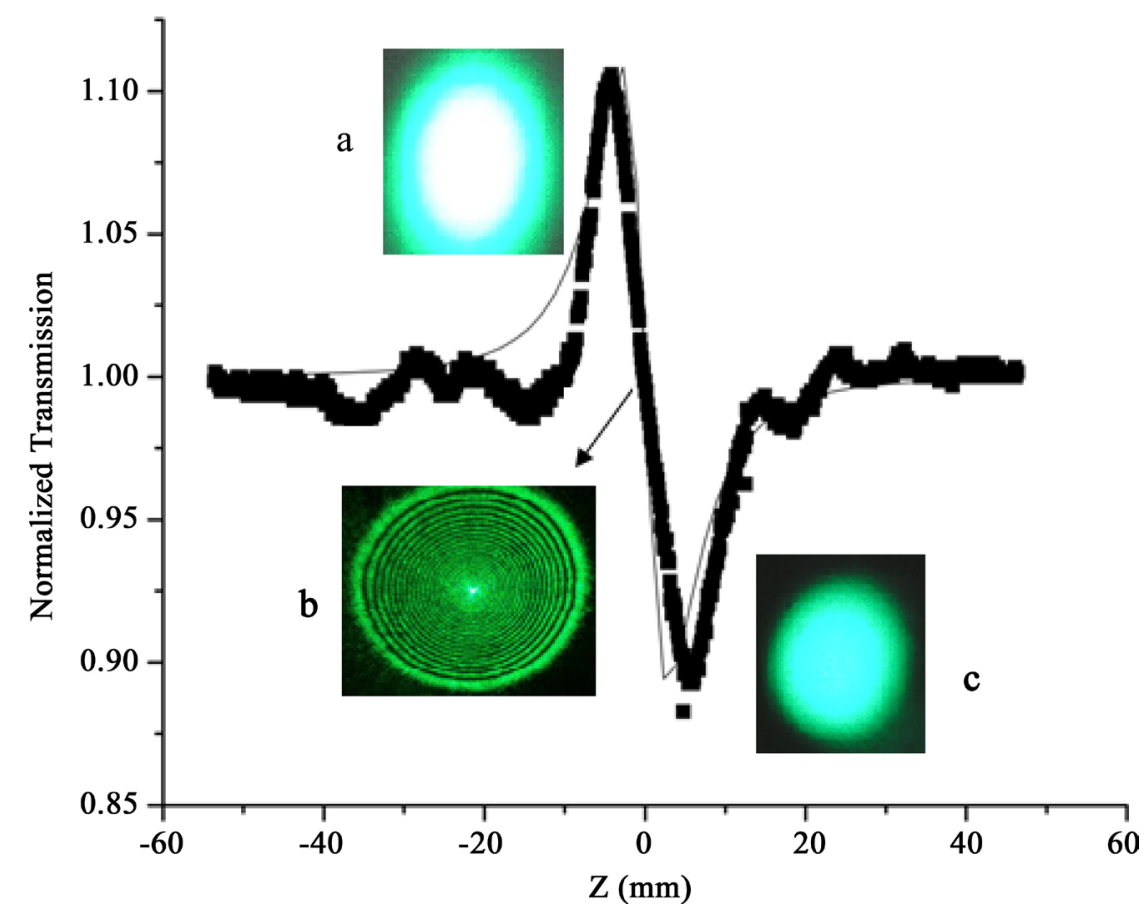

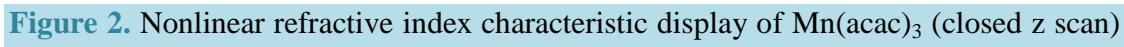
and the variation of the spot size as a function of the sample position. 
in the far field indicates the high value of nonlinear refractive index. Experiments are in progress to utilize the self-phase modulation technique for enhancing the contrast of biological imaging by employing the phase microscopy methods in a nonlinear regime.

Figure 3 shows the normalizing transmission for open aperture case. The nonlinear transmission is symmetric with respect to the focus $(\mathrm{z}=0)$, where it has minimum transmission. It is indicative that the sample exhibits reverse saturation absorption (RSA). RSA occurs when the absorption cross section of the excited states is larger than that of the absorption cross section of the ground state. The value for nonlinear absorption for Tris(acetylacetonato) Manganese(III) in solution is reported in [20].

The variation of intensities along the axis of the convex lens as the nonlinear sample passes through the focus can be used to simulate the intensities in different spatial frequency bands of the Fourier spectrum of the image. The spatial frequency distribution at the Fourier plane can be characterized into different intensity bands - low spatial frequencies at the center with high intensities and high spatial frequencies on the edges with low intensities. Thus the open z scan characteristics for example RSA can be exploited for its filtering property, which is slowly controllable with $\mathrm{z}$-positions ( low incident intensity away from the focus and maximum intensity at the focus $(\mathrm{z}=0)$ ). At low beam intensity, away from the focus position, no nonlinear effect is present, all the spatial frequencies are transmitted through the sample cell, without the filtering process. As the intensity increases, low frequencies begin to attenuate and they diminish at higher intensities. Thus the reverse saturation absorption effects for a given sample make it possible to calculate the required input intensities to obtain the desired band of spatial frequencies.

The transmission characteristics of Tris(acetylacetonato) Manganese(III) solution as a function of intensities (positions) are used to manipulate the spatial frequencies at the focal plane. Figure 3 (left) shows the results of edge enhancement of the inner portion of the grid which is used as the object. The corresponding position on the graph is marked as a function of position (intensity). At low input intensity, no nonlinear effect is present, the 4-f z scan setup will image the object on the screen without any spatial filtering (position a). As the sample moved closer to focus the intensity reaches adequate level to initiate nonlinearities in the sample and the image of the object start to diminish (position b). As the intensity increases above some threshold level (position c), low spatial frequencies start to disappear, resulting in the slow enhancement of edges with high spatial frequencies. As the intensity increases more low spatial frequencies are blocked, resulting in more enhancements of the edges. A nearly perfect edge enhancement is observed at position (d) (High intensity position).

Figure 4 shows the result of edge enhancement of a binary image placed at low intensity regions with no nonlinear effect present (a) and high intensity with nonlinear effect is present (b), Figure 4(a) represents original
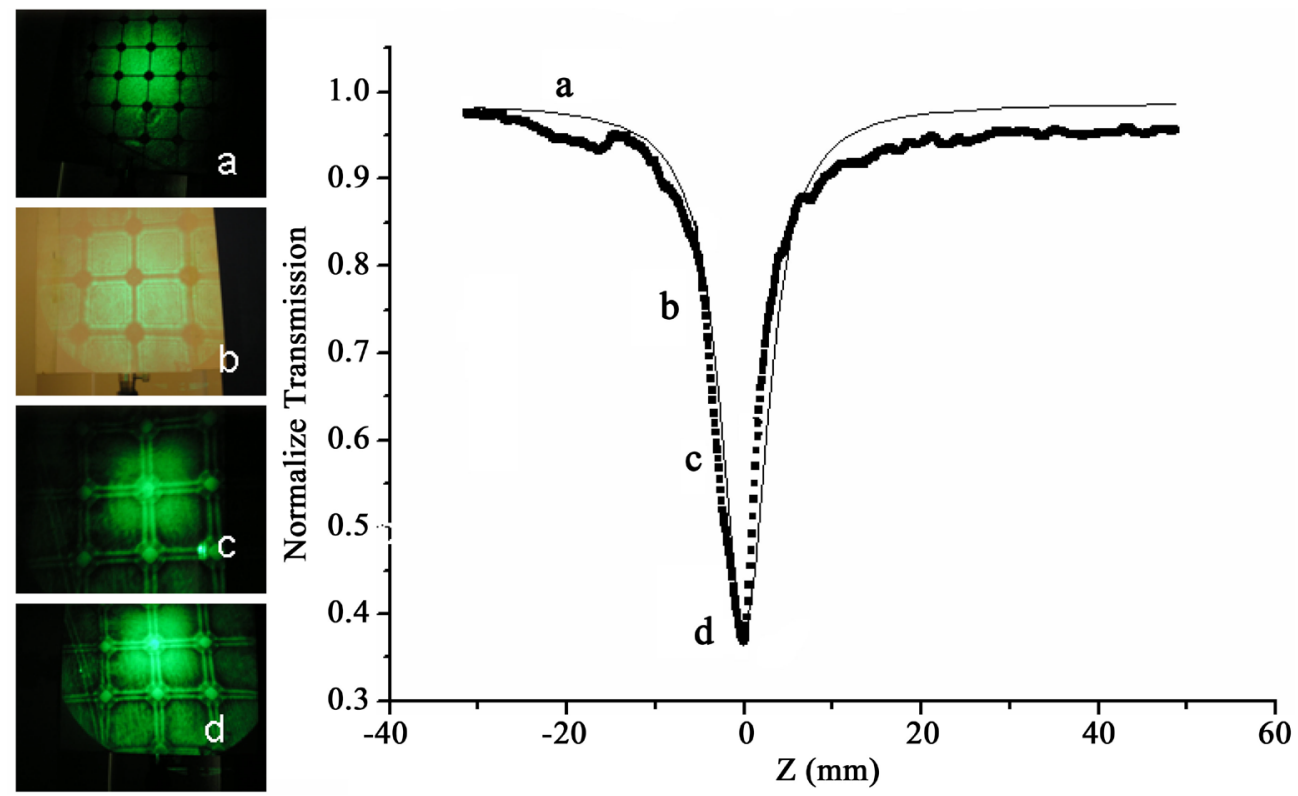

Figure 3. Nonlinear absorption characteristic display of Mn(acac) ${ }_{3}$ (open z scan) and processed images showing the edge enhancement. 
image without any spatial filtering and Figure 4(b) represents edge enhancement image. From above we conclude the technique is able to perform filtering process at the Fourier plane where the nonlinear material is placed. Different intensities give rise to different types of spatial frequency spectra and works for processing any complex frequency spectrum (any shape of object) without any preferential direction.

The technique is used to process blood samples for the imaging of red blood cells from normal individuals and sickle cell patients. The whole blood samples were obtained from Hematology laboratory Bahrain Defense Force (BDF) Hospital. A single drop of blood from each sample was placed on the microscope slide and left to dry for 24 hours. The blood samples were introduced as an object in the experimental setup. By controlling the input intensity entering the nonlinear sample at the Fourier plane where the nonlinear material $\left(\mathrm{Mn}(\mathrm{acac})_{3}\right)$ is placed, the edge enhancement of the blood samples was achieved. Figure 5(a) \& Figure 5(b) show the edge enhancement images of red blood cells for two normal individuals and Figure 5(c) for a sickle cell patient. The technique successfully used for imaging and edge enhancement of red blood cells and can easily be used to monitor the changes in the shape of blood cells.

\section{Conclusion}

In conclusion, the nonlinear characteristics of Tris(acetylacetonato) Manganese(III) was used to manipulate the spatial frequencies at the Fourier plane. The technique is a simple self-adaptive all-optical system, which performs image processing and nonlinear optical measurements at the same time. The technique is used in displaying
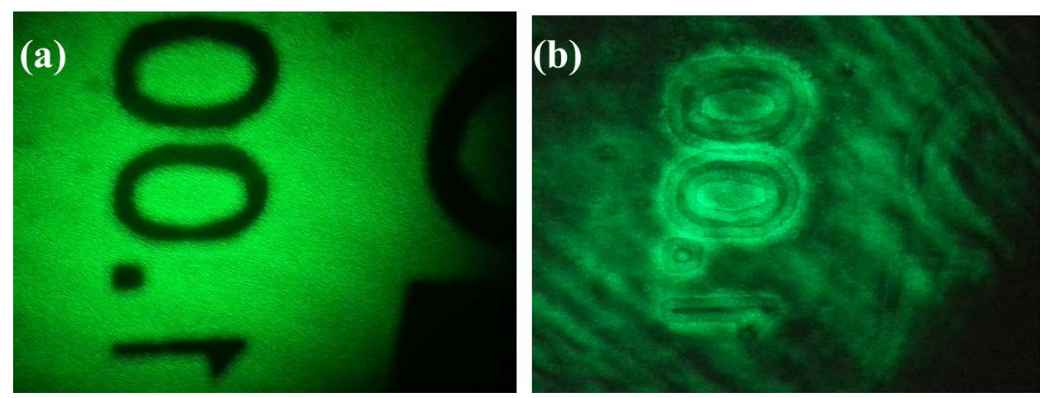

Figure 4. Processed images showing the edge enhancement (a) original object image at low intensity (b) edge enhancement image at high intensity.
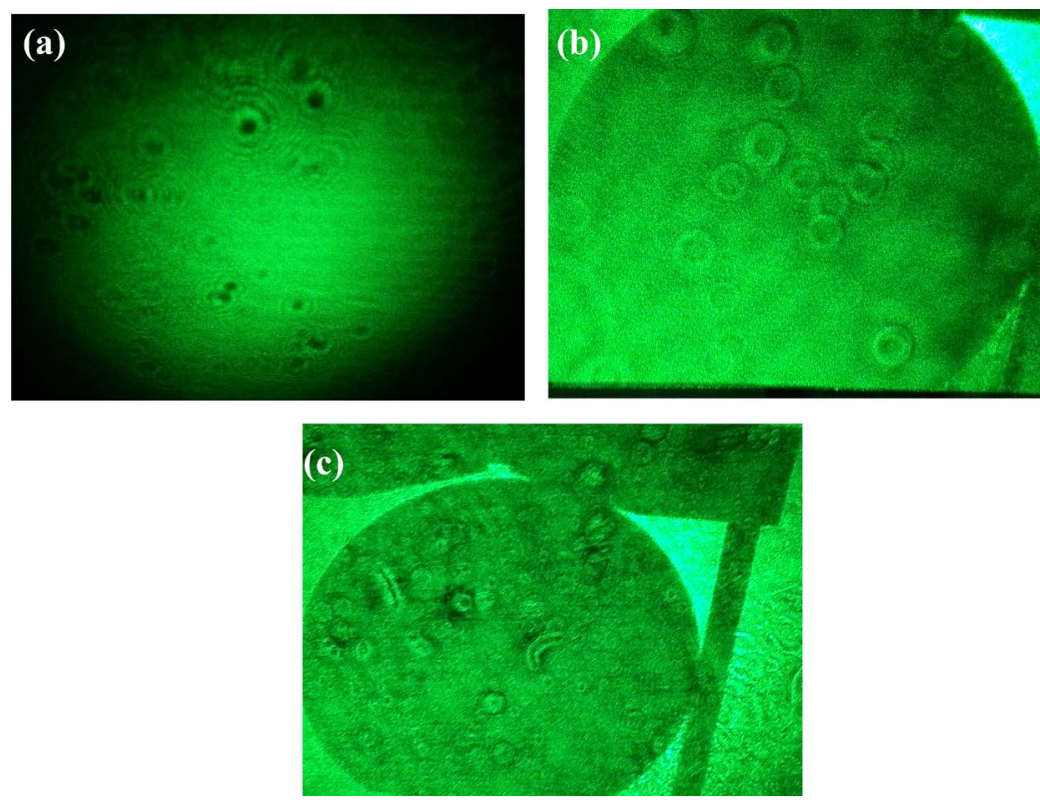

Figure 5. The edge enhancement images of red blood cells for two normal sample ((a), (b)) and (c) for sickle cell sample. The images were captured with digital camera. 
features of the red blood cells for normal individuals and for sickle cell patients, which opens the possibility of using the technique for sickle cell diagnosis. The technique also may be used for enhancing the visibility of film mammograms which may lead to early detection of microcalcification in breast cancer. The technique has a potential for optical implementation of image subtraction that can be utilized for medical image processing. Further experiments are in progress to investigate the use of this method for medical image processing.

\section{Acknowledgements}

This work is supported by RCSI-Medical University of Bahrain. The author would like to thank Dr. Seamus Cassidy for his help.

\section{References}

[1] Sheik-Bahae, M., Said, A.A., Wei, T.H., Hagan, D.J. and Van Stryland, E.W. (1990) Sensitive Measurement of Optical Nonlinearities Using Single Beam. IEEE Journal of Quantum Electronics, 26, 760-769. http://dx.doi.org/10.1109/3.53394

[2] Prasad, P.N. and Williams, D.J., Eds. (1991) Introduction to Nonlinear Optical Effects in Molecules and Polymers, John Wiley and Sons, New York.

[3] Jortener, J. and Ratner, M., Eds. (1997) Molecular Electronics. Blackwell Scientific, Oxford.

[4] Singh, C.P. and Roy, S. (2004) Dynamics of All-Optical Switching in C60 and Its Application to Optical Logic Gates. Optical Engineering, 43, 426-431. http://dx.doi.org/10.1117/1.1637905

[5] Chang, Y., Hong, J.H. and Yeh, P. (1990) Spatial Amplification: An Image-Processing Technique Using the Selective Amplification of Spatial Frequencies. Optics Letters, 15, 743-748. http://dx.doi.org/10.1364/OL.15.000743

[6] Yelleswarapu, C.S., Wu, P., Kothapalli, Sri-R. and Rae, D.V.G.L.N. (2006) All Optical Spatial Filtering with Power Limiting Materials. Optics Express, 14, 1451-1457. http://dx.doi.org/10.1364/OE.14.001451

[7] Sendhil, K., Vijayan, C. and Kothiyal, M.P. (2005) Spatial Phase Filtering with a Porphyrin Derivative as a Phase Filter in an Optical Image Processor. Optics Communications, 251, 292-298. http://dx.doi.org/10.1016/j.optcom.2005.03.014

[8] Nalwa, H.S. and Miyata, S., Eds. (1997) Nonlinear Optics of Organic Molecules and Polymers. Chemical Rubber Company, Boca Raton.

[9] Eichler, H.J., Günter, P. and Pohl, D.W. (1986) Laser-Induced Dynamic Gratings. Vol. 50 of Springer Series in Optical Sciences, Springer-Verlag, Berlin.

[10] Henari, F.Z. (2001) Optical Switching in Organometallic Phthalocyanine. Journal of Optics A: Pure and Applied Optics, 3, 188-190. http://dx.doi.org/10.1088/1464-4258/3/3/306

[11] Marder, S.R., Torruellas, W.E., Blanchard-Desce, M., Ricci, V., Stegeman, G.I., Gilmour, S., Bredas, J.-L., Li, J., Bublitz, G.U. and Boxer, S.G. (1997) Large Molecular Third-Order Optical Nonlinearities in Polarized Carotenoids. Science, 276, 1233-1236. http://dx.doi.org/10.1126/science.276.5316.1233

[12] Hache, A. and Bougeois, M. (2000) Ultrafast All Optical Switching in a Silicon Based Photonic Crystal. Applied Physics Letters, 25, 4089-4092. http://dx.doi.org/10.1063/1.1332823

[13] Henari, F.Z. and Jasim, K.E. (2013) All Optical Switching in Hena thin Film. Applied Physics B, 112, 261-265. http://dx.doi.org/10.1007/s00340-013-5429-y

[14] Yisoon, B., Haus, J.W., Scalora, M. and Sibilia, C. (2003) One-Dimensional Photonic Crystal Optical Limiter. Optics Express, 11, 2007-2018. http://dx.doi.org/10.1364/OE.11.002007

[15] Goodman, J.W. (1968) Introduction to Fourier Optics. McGraw-Hill, San Francisco, CA.

[16] Yelleswarapu, C.S., Kothapalli, Sri-R. and Rae, D.V.G.L.N. (2008) Optical Fourier Techniques for Medical Image Processing and Phase Contrast Imaging. Optics Communications, 281, 1876-1888. http://dx.doi.org/10.1016/j.optcom.2007.05.072

[17] Phu Xuan, Ng., Ferrier, J.L., Gazengel, J., Rivoire, G., Brekhovskikh, G.L., Kudryavtseva, A.D., Sokolovskaya, A.I. and Tcherniega, N.V. (1988) Changes in the Structures of Light Beams Induced by Nonlinear Optical Phenomena: Application to Phase Contrast and Image Processing. Optics Communications, 68, 244-249. http://dx.doi.org/10.1016/0030-4018(88)90393-8

[18] Shih, M.S., Shishido, A. and Khoo, I.C. (2001) All-Optical Image Processing by Means of a Photosensitive Nonlinear Liquid-Crystal Film: Edge Enhancement and Image Addition-Subtraction. Optics Letters, 26, 1140-1142. http://dx.doi.org/10.1364/OL.26.001140 
[19] Joseph, J., Aranda, F.J., Rao, D.V.G.L.N., Akkara, J.A. and Nakashima, M. (1966) Optical Fourier Processing Using Photo induced Dichroism in a Bacteriorhodospin Film. Optics Letters, 22, 1499-1501.

[20] Henari, F.Z. and Ali Mohamed, A.Y. (2008) Nonlinear Refractive Index Measurement of Tris(acetylacetonato) Manganese(III) Solution. Optics \& Laser Technology, 40, 602-606. http://dx.doi.org/10.1016/j.optlastec.2007.09.005

\section{Submit or recommend next manuscript to SCIRP and we will provide best service for you:}

Accepting pre-submission inquiries through Email, Facebook, Linkedin, Twitter, etc A wide selection of journals (inclusive of 9 subjects, more than 200 journals)

Providing a 24-hour high-quality service

User-friendly online submission system

Fair and swift peer-review system

Efficient typesetting and proofreading procedure

Display of the result of downloads and visits, as well as the number of cited articles

Maximum dissemination of your research work

Submit your manuscript at: http://papersubmission.scirp.org/ 